\title{
Analyses of pathological cranial ultrasound findings in neonates that fall outside recent indication guidelines: results of a population-based birth cohort: survey of neonates in Pommerania (SNiP-study)
}

Judith Weise', Matthias Heckmann', Hagen Bahlmann', Till Ittermann², Heike Allenberg' ${ }^{1}$, Grzegorz Domanski ${ }^{1}$ and Anja Erika Lange ${ }^{1 *}$

\begin{abstract}
Background: Recent guidelines recommend a cranial ultrasound (CU) in neonates born at $<30$ weeks gestation, admitted to the neonatal intensive care unit (NICU), or with a CU indication. Here, we addressed the need to extend these recommendations.

Methods: We retrospectively reviewed 5107 CUs acquired in the population-based Survey of Neonates in Pomerania, conducted in 2002 to 2008. Neonates with conspicuous CUs that were $\geq 30$ weeks gestation without recent indications for CU were identified and assigned to the following groups: with (I) or without (II) admission to neonatal care. We designated CU conspicuities as mild (MC) or significant (SC), and we investigated related neurodevelopment during follow-up.

Results: Of 5107 neonates, 5064 were born at $\geq 30$ weeks gestation and of those, 4306 received CUs without any indication for this examination. We found conspicuities in 7.7\% ( $n=47 / 610)$ of group I ( $n=30 \mathrm{MC}, n=17 \mathrm{SC})$, and $3.2 \%(n=117 / 3696)$ of group II ( $n=100 \mathrm{MC}, \mathrm{n}=17 \mathrm{SC}$ ). In group II, SC comprised, e.g., bilateral cysts, partial agenesis of the corpus callosum, and periventricular leukomalacia. Follow-up was available in $75 \%$ of infants in group II with MCs and SCs; of these, 12.8\% had an abnormal neurological follow-up.

Conclusions: We detected a high number of conspicuities in neonates without a CU indication. However, we could not demonstrate that ultrasound findings were associated with the neurological follow-up or any advantage to an earlier diagnosis. Our data did not support extending current guidelines or a general CU screening policy for all neonates.
\end{abstract}

\section{Background}

The cranial ultrasound (CU) is a cost-effective, portable, non-invasive examination that does not require radiation. These features make it a valuable tool for assessing infantile brain structures [1]. The CU can detect cerebral pathologies, like hemorrhages or ventricle system disorders, without sedation [2]. Furthermore, early

\footnotetext{
* Correspondence: anja.lange@uni-greifswald.de

${ }^{1}$ Dept. of Neonatology \& Paediatric Intensive Care Medicine, University

Greifswald, F.-Sauerbruchstr, 17475 Greifswald, Germany

Full list of author information is available at the end of the article
}

detection of these lesions ensures appropriate medical management and long-term assessments of neurodevelopmental disabilities [3].

Currently, the Quality Standards Subcommittee of the American Academy of Neurology and the Practice Committee of the Child Neurology Science recommend routine CU screening for all neonates $<30$ weeks of gestational age (weeks GA) [3]. Beyond those recommendations, Leijser et al. claimed to include neonates that were admitted to the neonatal care unit $(\mathrm{NCU})$ after birth [4]. The American Institute of Ultrasound in

(c) The Author(s). 2019 Open Access This article is distributed under the terms of the Creative Commons Attribution 4.0 International License (http://creativecommons.org/licenses/by/4.0/), which permits unrestricted use, distribution, and reproduction in any medium, provided you give appropriate credit to the original author(s) and the source, provide a link to the Creative Commons license, and indicate if changes were made. The Creative Commons Public Domain Dedication waiver (http://creativecommons.org/publicdomain/zero/1.0/) applies to the data made available in this article, unless otherwise stated. 
Table 1 Established AIUM indications for a neonatal CU and corresponding variables as predictors measured in the SNiP study [5]

\begin{tabular}{|c|c|}
\hline AlUM Indications for CU in neonates & corresponding variables in the SNiP study \\
\hline $\begin{array}{l}\text { Hemorrhage or parenchymal abnormalities in } \\
\text { preterm and term infants }\end{array}$ & $\begin{array}{l}\text { birthweight }<1500 \mathrm{~g} \text { and }<30 \mathrm{WG}[3] \text {, vacuum or forceps delivery [8], systemic hypertension or } \\
\text { hypotension, or perinatal asphyxia [9] }\end{array}$ \\
\hline Hydrocephalus & signs (e.g., macrocephaly, curved fontanel, split cranial sutures) [10] \\
\hline Vascular abnormalities & prenatal diagnosis (e.g., vascular malformations, aneurysms) \\
\hline $\begin{array}{l}\text { Possible or suspected hypoxic ischemic } \\
\text { encephalopathy }\end{array}$ & $\begin{array}{l}\text { symptoms (e.g., seizures, hypotonia, coma, respiratory distress), UA pH }<7.0 \text {, APGAR value of } 0 \text { - } \\
3 \text { at }>5 \text { min [3] }\end{array}$ \\
\hline Congenital malformations & $\begin{array}{l}\text { prenatal diagnoses (e.g., cysts, cerebellar hypoplasia) [11], chromosomal anomalies or } \\
\text { malformations, or metabolic diseases [12] }\end{array}$ \\
\hline Congenital or acquired brain infections & mycoplasma, toxoplasmosis, cytomegalovirus, streptococcus \\
\hline $\begin{array}{l}\text { Signs and/or symptoms of central nervous system } \\
\text { disorders }\end{array}$ & $\begin{array}{l}\text { symptoms (e.g., facial malformations, macrocephaly, microcephaly, intrauterine growth } \\
\text { restriction) [5] }\end{array}$ \\
\hline Trauma & cephalo/subgaleal hematoma, subdural hematoma, subarachnoidal hemorrhage \\
\hline Craniosynostosis & craniosynostosis \\
\hline $\begin{array}{l}\text { Previously documented abnormalities, including } \\
\text { prenatal abnormalities }\end{array}$ & prenatal diagnoses (e.g., partial/complete agenesis of corpus callosum) \\
\hline $\begin{array}{l}\text { Patients treated with hypothermia, ECMO, or other } \\
\text { support systems }\end{array}$ & hypothermia, ECMO etc. \\
\hline
\end{tabular}

Medicine (AIUM) Practice Guidelines for the Performance of Neurosonography in Neonates and Infants 2014 also recommend $\mathrm{CU}$ for all infants with indications (Table 1) [5]. In Germany, neonatal screening for the auditory system, hip dysplasia, and metabolic diseases, but not CU, are included in regular check-ups ("U2/U3") to ensure the early detection of treatable diseases with severe outcomes.

Additionally, assessments of fetal brain structures and possible deviations could be conducted with a prenatal ultrasound. Like CU, this prenatal examination is not generally performed in Germany, but recently, it has been offered regularly in the study region in western Pomerania.

The population-based birth cohort study, known as the Survey of Neonates in Pomerania (SNiP), included a $\mathrm{CU}$ for all participants, irrespective of indication, gestational age, or inpatient admission [6]. Here, we retrospectively reviewed those data. We aimed to determine the prevalence, severity, and outcome of cerebral conspicuities diagnosed in neonates, when there was no CU indication, according to conventional guidelines; i.e., we addressed the need for an extension of recent $\mathrm{CU}$ recommendations.

\section{Methods}

\section{Study design}

For the present analysis, we acquired data from the population-based prospective birth cohort study, SNiP, from 2002 to 2008 collecting data about morbidity and mortality of newborns and their mothers in a predefined region yielding to data about prevalence, risk factors and confounders for/of neonatal diseases. Based on this, cranial ultrasound was used to assess potential cerebral disorders. Physicians trained for this study collected data on newborn children and their mothers, regarding neonatal health, morbidity, and mortality. We calculated the prevalence rates for major neonatal diseases, risk factors, and confounding conditions, on a cross-sectional, prospective basis. All mothers with a complete data set were included, and they provided written informed consent to participate in the study.

The data collection was conducted as follows: (i) a standardized 5 to 10 -min interview (84 variables), (ii) a self-administered questionnaire concerning socioeconomic background (40 variables), and (iii) a review of maternal medical records and the prenatal care booklet concerning the gestational period and any preventive examinations (149 variables). Details of the SNiP study have been reported previously by Ebner et al. [6] The collected data were anonymized and stored in an Access database. Approval for this study was obtained from the Ethics Committee of the Medical School of University. All analyses were based on complete data sets.

\section{Population}

Current CU recommendations and the AIUM Practice Guidelines 2014 include indications: gestational age $<30$ weeks GA, several diseases with correlation to neurodevelopmental disorders (see Table 1 ) $[3,5]$. To evaluate the prevalence and severity of diagnoses in infants that fell outside the recent recommendations for $\mathrm{CU}$, we 
identified all neonates with $\mathrm{CU}$ conspicuities that were born $\geq 30$ weeks GA without indication, and grouped them as follows: (group I) neonates with admission and (group II) without admission to NCU after birth [5]. With respect to severity of illness, or degree of immaturity sick neonates were admitted to the neonatal care unit (special care baby unit or low dependency unit) to the intensive care unit (NICU). However, it was not possible to distinguish between these within the SNiP database. In general, all newborns of less than 34 weeks GA were admitted to special or intensive neonatal care based on severity of disease. Neonates $\geq 30$ weeks GA with conspicuous lesions on the CU were additionally grouped by gestational maturity, as very/moderately preterm (30 to 34 weeks GA, $n=129$ ) or late preterm/term (>34 weeks GA, $n=4937$ ).

\section{Examinations}

The SNiP CU protocol was differentiated, with respect to weeks GA and admission: (i) CUs were conducted on days $1,3,7$, and every 14 days for all neonates born $<30$ weeks GA; (ii) CUs were conducted between the 30th and 36th weeks GA for neonates admitted to the NCU; and (iii) CUs were conducted between the 3rd and 10th day of life (check-up "E2") for all other neonates. Consultants and registrars at the participating hospitals in Greifswald performed the CUs with a Sonolayer SSA-270A (TOSHIBA Medical Systems, Otawara, Japan), Acuson 128XP/10 Ultrasound System (Acuson, Mountain View, CA), the registrars at the participant hospital in Wolgast Picker CS 9100 EVB-405 (Bra Medik, HITACHI), and in Anklam Logia TM 200 (Pro Serius, Bangalore, India), and a 7.5 $\mathrm{MHz}$ sector array transducer; e.g., Wi Pro (GE Medical Systems, Bangalore, India). Afterwards, the findings were controlled by consultants or chief residents for Neonatology according to the hospital standards of University hospital, Greifswald. All CUs were performed in accordance with German performance guidelines [7].

An intrauterine ultrasound between the 19th to 22nd week of gestation was offered to all participants by German health insurance groups, as part of a prenatal prevention program, searching for malformations and especially brain abnormalities, which yielded to e.g. changes of the biparietal, frontooccipital diameter or head circumference. Next to this the prenatal prevention program contented ultrasounds between the 9th to 12th and 29th to 32nd week of gestation. Furthermore, all neonates were examined neurologically to evaluate tone, reflexes, and posture, during two regular check-ups ("U1/U2") within the first 10 days of life. These examinations were not part of the SNiP protocol, but results were recorded in the SNiP database. When cerebral conspicuities were detected, the parents were given information about the necessity of a follow-up and the potential long-term outcome of the conspicuities found.

\section{Analyses}

Associated risk factors, signs, and symptoms that were considered indications for $\mathrm{CU}$, according to the AIUM Practice Guidelines, were related to variables in the SNiP database (Table 1). This procedure yielded the preanalysis of neonates with indication for $\mathrm{CU}$ and classification into groups I and II.

For this study, all CU images with pathological findings were re-evaluated by one experienced neonatologist (A.E.L.). Pathological findings were classified as mild (MC) or significant (SC) conspicuities (Table 2). Furthermore, normal variants or undocumented cases in patient's file or SNiP data base, which were marked as conspicuous in the primary database, could be excluded in this step to reduce the false positive rate of abnormal findings. We assessed long-term outcomes of neonates with MCs and SCs in the CUs. The University hospital, Greifswald is the only location in the study region with specialists for pediatric neurology and diagnostic and therapeutic interventions for neurodevelopmental disorders. When possible, we searched the medical records until recent days, with the following keywords: delay or disorder of motor and language development, learning disorders, hemiplegia, mental retardation, cerebral palsy, epilepsy, or diagnosis-related neonatal death. We analyzed records of neonates without a recent indication for $\mathrm{CU}$ or a NCU admission to investigate significant correlations with the following pre-, peri-, and postnatal predictors: infection with mycoplasma, streptococci, toxoplasmosis or cytomegalovirus, steroid administration in pregnancy for prevention of respiratory distress syndrome, chronic maternal condition, vacuum or forceps delivery, birth risks, APGAR score $<7$ at 5 min (assessment score for appearance, pulse, grimace, activity, and respiration of the neonate), arterial umbilical cord blood $\mathrm{pH}<7.0,<10$ th or $>90$ th percentile birth weight, microor macrocephalus, postnatal hypoglycemia, infection (e.g., Escherichia. coli), or respiratory distress.

\section{Statistics}

All analyses were performed with SPSS $^{\bullet}$ version 22 for Windows $7 / 10^{\circ}$ (Microsoft Corporation). We used the Fisher's exact test for comparing $3 \times 3$-tables for statistical significance, which was set to a $p$-value $<0.05$. Continuous data were expressed as the median to avoid excess influence from extreme values $(<$ or $>95 \%$ of a normal curve). Categorical data were described with absolute numbers and percentages.

\section{Results}

\section{Cranial ultrasound in the study population}

In total, 7220 neonates were born in the study region during the evaluated period. Of these, 70.7\% $(n=5107$, median gestational age 39 weeks, median birth weight 
Table 2 Classification of CU findings into significant or mild conspicuities, according to pathological neurodevelopmental sequelae

\begin{tabular}{|c|c|}
\hline Cranial ultrasound & Neurodevelopmental outcome in the literature \\
\hline \multicolumn{2}{|l|}{ Significant conspicuities } \\
\hline Corpus callosum malformation & $\begin{array}{l}\text { Mild behavioral problems to severe neurological disorders (e.g., autistic } \\
\text { behavior), associated genetic syndromes, aneuploidies, malformations, } \\
\text { inborn errors of metabolism [13-15] }\end{array}$ \\
\hline $\begin{array}{l}\text { Bilateral/multiple cyst } \\
(2-5 \text { bilateral and/or unilateral cysts (max. } 0.3-1.5 \mathrm{~cm}))\end{array}$ & Congenital infection or genetic anomaly [16] \\
\hline $\mathrm{IVH}, \mathrm{II}-\mathrm{IV}^{\circ}$ & $\begin{array}{l}\text { Epileptic disorders, perceptual difficulties, cognitive deficiencies, mental } \\
\text { handicaps }[10,16,17]\end{array}$ \\
\hline Hydrocephalus & $\begin{array}{l}\text { Associated congenital brain anomalies, post-hemorrhagic, infection with } \\
\text { neuromotor disorders, hearing loss, blindness, epilepsy }[18,19]\end{array}$ \\
\hline $\begin{array}{l}\text { Periventricular leukomalacia (> } 5 \text { cysts }>0.3 \mathrm{~cm} \text {, along the corpus } \\
\text { callosum) }\end{array}$ & $\begin{array}{l}\text { Global delay in myelination correlated with cerebral palsy and cognitive/ } \\
\text { behavioral abnormalities }[10,20]\end{array}$ \\
\hline \multicolumn{2}{|l|}{ Mild conspicuities } \\
\hline $\begin{array}{l}\text { Ventricular asymmetry/enlargement } \\
(>0.5 \mathrm{~cm} \text { difference of vertical distance between sulcus thalamicus and } \\
\text { corpus callosum in two sagittal views at the level of the plexus } \\
\text { choroideus in the lateral ventricles) }\end{array}$ & $\begin{array}{l}\text { Normal variants or variants associated with autism, attention deficit } \\
\text { hyperactivity disorder, learning disorders }[22,23,21]\end{array}$ \\
\hline Unilateral/singular cyst & No screening necessary [16] \\
\hline Increasing echogenicity & $\begin{array}{l}\text { Physiologic immaturity of myelination of preterm infants or associated } \\
\text { with hemorrhages, edemas etc. (follow-up necessary) [19] }\end{array}$ \\
\hline $\mathrm{IVH} \mathrm{I}^{\circ}$ & No increase of conspicuous neurological impairment [18] \\
\hline
\end{tabular}

CU: cranial ultrasound, IVH: intraventricular hemorrhage

$3420 \mathrm{~g}$ ) were included in the SNiP study (Fig. 1). Of these, 5107 infants received CUs, regardless of indication, gestational age, or admission. Error! Reference source not found. Shows the examined population according to predefined groups.

\section{Frequency of cranial ultrasound findings and classifications}

In the primary database, 339/5107 (6.6\%) ultrasound findings were classified as conspicuous. A re-evaluation of the stored ultrasound images was necessary to exclude normal variations, like Cavum septum pellucidum, to detect undocumented cases, and to assign conspicuities as either mild (MC) or significant (SC). This evaluation led to a reduction in the number of conspicuities (final $n=237 ; 4.6 \%$ ) in the study population. To assess whether recent guidelines should be extended, we exclusively analyzed neonates born $\geq 30$ weeks GA and without indication for $\mathrm{CU}(n=4306)$.

758 neonates $\geq 30$ weeks GA had indication for CU, based on AIUM Practice Guidelines. Of these, $n=58$ (7.65\%) had conspicuities in the CU.

4306 neonates had no indication for CU. Of these, 140 (3.3\%) had a conspicuous CU (81.4\% MC and $18.6 \%$ SC; Fig. 1, Table 3). Among 610 neonates without indication for CU but who were admitted to the NCU (group I), 7.7\% ( $n=47)$ had conspicuities in the CU. Among 3696 neonates with no indication who were not admitted to the NCU (group II), 3.2\% ( $n=117)$ had conspicuities in the CU. Details of MC are given in Table 4 and details of SC are given in Table 5 (Table 4, Table 5). All neonates with significant conspicuous $\mathrm{CU}$ had inconspicuous prenatal ultrasounds between the 19th and 22nd week. All neonates with significant conspicuous CU had controlled per MRI brain scan.

\section{Risk factors for pathologic findings in $\mathrm{CU}$}

The analysis of pre-, peri- and post-natal risk factors for conspicuous $\mathrm{CU}$ in neonates without a NCU admission or an indication for $\mathrm{CU}$ (II) yielded no significant risk factors for this group. Documented cases of named predictors are shown in Table 6 (Table 6).

\section{Long-term follow-up after conspicuous cerebral ultrasound}

We investigated the documentation of neurodevelopmental follow-ups for all neonates with conspicuous CUs. These patients received at least a second CU scan to assess progression, stability, or regression of MCs and SCs. Of 140 neonates, 99 were further seen, examined or treated by the medical staff of the University Hospital, Greifswald (missing data group I: $n=12 / 47$; group II: $n=29 / 117$ ). Of the 99 patients monitored, 17 with MCs and 7 with SCs had the necessity of neurodevelopmental follow-ups (group I: $n=4 \mathrm{MCs}, n=5 \mathrm{SCs}$; group II: $n=$ $13 \mathrm{MCs}, \mathrm{n}=2 \mathrm{SCs}$ ). A significantly higher proportion of neurodevelopmental disorders was found among neonates with SCs $(23.1 \%)$ compared to those with MCs (9.7\%; $p<0.05$ in group I and II). Of all neonates with admission to NCU (group I), 14.3\% $(n=6 / 42)$ had a 


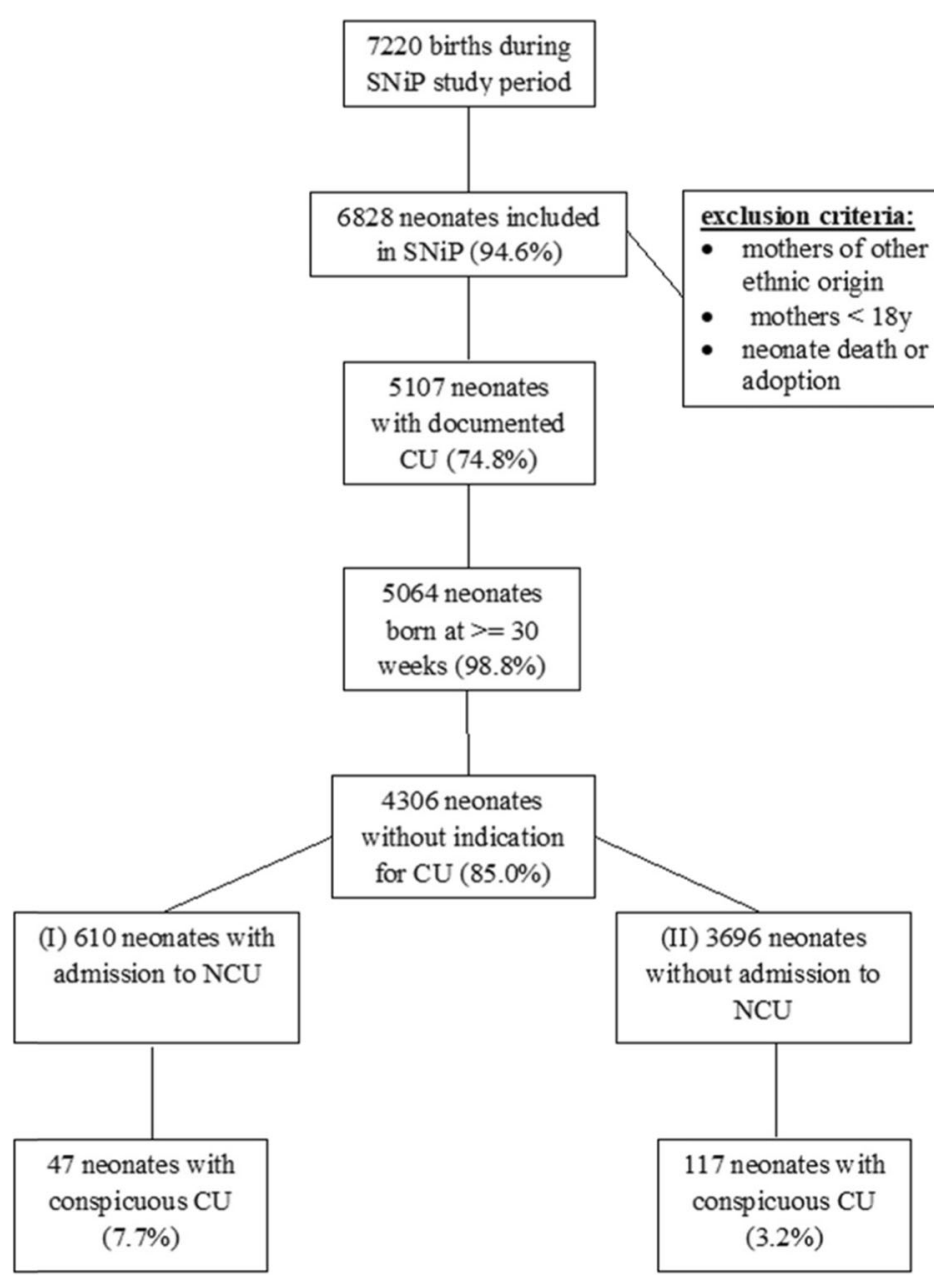

Fig. 1 Flow chart of patient inclusion and grouping for analysis. SNiP: Survey of Neonates in Pomerania; CU: cerebral ultrasound; NCU: neonatal care unit; conspicuous CU: a potential lesion detected on the CU

Table 3 Frequency of conspicuous cranial ultrasound findings in neonates $>=30$ weeks of gestation without indication for cranial ultrasound

\begin{tabular}{lc}
\hline Cranial ultrasound groups & N of subgroup n/ N of group (\%) \\
\hline Conspicuities detected & $140 / 3809(3.7)$ \\
at ages 30-34 weeks gestation & $4 / 12(33.3)$ \\
at ages > 34 weeks gestation & $136 / 3797(3.6)$ \\
AND NCU admission (group I) & $47 / 610(7.7)$ \\
mild & $30(4.9)$ \\
significant & $17(2.8)$ \\
WITHOUT NCU admission (group II) & $117 / 3696(3.2)$ \\
mild & $100(2.7)$ \\
significant & $17(0.5)$
\end{tabular}


Table 4 Frequency of mild conspicuities on cerebral ultrasound distributed among different gestational ages and groups

\begin{tabular}{lll}
\hline $\begin{array}{l}\text { Gestational age group } \\
\text { type of conspicuity }\end{array}$ & $\begin{array}{l}\text { Group I } \\
\text { Total cases }\end{array}$ & Group II \\
\hline 30-34 weeks GA & $\mathbf{3}$ & - \\
unilateral singular cyst & 2 & \\
ventricular enlargement/asymmetry* & 1 & $\mathbf{1 0 0}$ \\
$>$ 34 weeks GA & $\mathbf{2 7}$ & 55 \\
ventricular enlargement/asymmetry* & 12 & 38 \\
unilateral singular parenchymal cyst & 9 & 4 \\
IVH I & 1 & 3 \\
increased echogenicity & 5 & 1 \\
caudothalamic groove & & 1 \\
thalamic & & 1 \\
paraventricular & 4 & \\
frontotemporal & 1 &
\end{tabular}

Data are the number of infants in each group. Weeks GA: weeks of gestational age; IVH: intraventricular hemorrhage, *measurement: $>0.5 \mathrm{~cm}$ vertical distance difference between the sulcus thalamicus and the corpus callosum in two sagittal views at the level of the plexus choroideus in the lateral ventricles

neurodevelopmental disorder. Neurodevelopmental disorders were found in $11.5 \%(n=15 / 117)$ of patients in group II, and the cumulative incidence was $0.4 \%$ (Table 7). All of these neonates had negative microbiological blood tests for mycoplasma, cytomegalovirus, streptococcus, and toxoplasmosis. Neonates with partial agenesis of the corpus callosum $(\mathrm{n}=1)$ and intraventricular hemorrhage $\mathrm{II}^{\circ}(\mathrm{IVH} ; \mathrm{n}=2)$ were born with normal birth weights and APGAR scores. These three neonates showed no neurodevelopmental impairment related to a conspicuous CU during follow-up.

\section{Discussion}

Based on recent international guidelines, a neonatal $\mathrm{CU}$ is recommended for the following defined risk neonatal groups: aged $<30$ weeks GA or an indication for CU [3, 5]. Additionally, a proposal was made for neonates with admission to NCU [4]. In this study, we re-evaluated these recommendations to determine whether an extension was warranted. Specifically, we addressed whether performing a $\mathrm{CU}$ was justified for all infants, irrespective of indications, based on the prevalence and severity of $\mathrm{CU}$ conspicuities and their neurodevelopmental outcomes. Our population comprised 3696 neonates that had no indication for $\mathrm{CU}$ and were not admitted to the NCU. We found that the cumulative incidence of a conspicuous CU finding in neonates without an indication or NCU admission was $3.2 \%$, which was, as expected, lower than the incidences among neonates with indications (7.65\%) and neonates with NCU admissions (7.7\%). Significant anomalies, like corpus callosum hypoplasia, periventricular leukomalacia (PVL), and $\mathrm{IVH}^{\mathrm{I}}{ }^{\circ}$, were not associated with a conspicuous neurological follow-up; thus, we lacked a correlation between conspicuous $\mathrm{CU}$ findings and neurological impairments $[8,9]$. Among the 117 neonates without an indication for $\mathrm{CU}$, but with $\mathrm{CU}$ conspicuities, $85.5 \%$ had MCs and $14.5 \%$ had SCs. Ballardini et al. postulated a prevalence of $1.7 \%$ for conspicuous CUs in asymptomatic neonates aged $>37$ weeks $\mathrm{GA}$, and the distribution of MCs and SCs was similar to that in the present study [10]. The prevalence of SCs was $0.33 \%$ in group II, which was higher than the prevalence of significant anomalies found by Ballardini et al. $(0.19 \%)$ or that found by Wang et al. $(0.25 \%)$ $[10,11]$. The inclusion of bilateral cysts as an SC yielded a higher prevalence in our study. Even higher prevalences were reported by Heibel et al. (9\%) and Gover et al. (11.2\%). Those values might be explained by differences in operators, populations, and/ or techniques $[8,12]$. None of the neonates with bilateral cysts had positive microbiological blood tests. Therefore, this study could not demonstrate that the probability of congenital infections increased with the presence of bilateral parenchymal cysts [8].

Table 5 Frequency of significant conspicuities on cerebral ultrasound distributed among different gestational age and groups

\begin{tabular}{lll}
\hline $\begin{array}{l}\text { Gestational age group } \\
\text { type of conspicuity }\end{array}$ & Group I & Group II \\
\hline $30-34$ weeks GA & Total cases & - \\
bilateral/multiple cyst & 1 & 1 \\
$>34$ weeks GA & 16 & 13 \\
bilateral/multiple cyst & 8 & 2 \\
IVH II & 2 & 1 \\
PVL & 3 & 1 \\
partial agenesis of corpus callosum & 1 & 1 \\
Arnold-Chiari malformation type I & 1 &
\end{tabular}

Data are the number of infants in each group. Weeks GA: weeks of gestational age; IVH: intraventricular hemorrhage; PVL: periventricular leukomalacia, with $>5$ cysts $>0.3-\mathrm{cm}$ in diameter, along the corpus callosum; bilateral/multiple cyst: $2-5$ bilateral and/or unilateral cysts (max. $0.3-1.5 \mathrm{~cm}$ in diameter) [20]. 
Table 6 Univariate Analysis of potential predictors of conspicuities detected in cranial ultrasounds performed in neonates without NCU admission or indication for CU (Group II)

\begin{tabular}{|c|c|c|c|c|}
\hline Potential predictor & All cases $(n=3696)$ & Cases with mild conspicuities $(n=100)$ & Cases with significant conspicuities $(n=17)$ & $p$ value* \\
\hline APGAR $<7$ at $>5 \mathrm{~min}$ & $6(0.2)$ & $1(1.0)$ & 0 & 0.176 \\
\hline birth risks & $1147(31.0)$ & $35(35.0)$ & $5(29.4)$ & 0.671 \\
\hline birth weight & & & & 0.163 \\
\hline$<$ 10th percentile & $111(3.0)$ & $1(1.0)$ & 0 & \\
\hline$>90$ th percentile & $182(4.9)$ & $4(4.0)$ & $3(17.6)$ & \\
\hline \multicolumn{5}{|l|}{ delivery mode } \\
\hline spontaneous & $3297(89.2)$ & $88(88.0)$ & $14(82.4)$ & \\
\hline missed & $399(10.8)$ & $12(12.0)$ & $3(17.6)$ & \\
\hline maternal chron. Disease & $1154(31.2)$ & $38(38.0)$ & $5(29.4)$ & 0.133 \\
\hline RDS prophylaxis & $109(2.9)$ & $6(6.0)$ & 0 & 0.200 \\
\hline
\end{tabular}

Data are the number of individuals (\%) in each group. *Mild or significant conspicuities were compared to inconspicuous cranial ultrasounds. NCU: neonatal care unit; CU: cranial ultrasound; APGAR score: assessment score for appearance, pulse, grimace, activity, and respiration of the neonate; RDS: respiratory distress syndrome. Birth risks (based on documentation in German maternal booklet for prenatal examinations): e.g., multiple births, vaginal infections, twin births etc. $P$ values were derived with Fisher's Exact Test

Most MCs (93\%) in group II comprised ventricular asymmetry, mild ventricular enlargement, or unilateral singular cysts. Of these MCs, $13 \%(n=13 / 100)$ were associated with later neurological impairments, like attention deficit (and hyperactivity) disorder, speaking/ language disorders, or epilepsy. This finding did not suggest that the guidelines should be extended for these diagnoses, because, for example, single cysts had a negligible likelihood of leading to neurodevelopmental impairment, and thus, they did not require follow-up [8].A large proportion of SCs (76\%) in group II comprised bilateral or multiple cysts. These cysts can be diagnosed easily with CU. One in 4-5 neonates with bilateral, multiple choroid plexus cysts or subependymal pseudocysts harbor a chromosomal anomaly, like trisomy 18 , trisomy 21 , Cri du chat syndrome, or Zellweger syndrome, or a congenital infection (cytomegalovirus or rubella virus) with the highest positive likelihood ratio

Table 7 Neurodevelopmental outcome in neonates with significant $(n=2)$ and mild $(n=13)$ conspicuities detected in the CU, despite no AlUM indication or NCU admission (group II)

\begin{tabular}{|c|c|c|c|c|c|}
\hline Case & $\begin{array}{l}\text { Gestational age } \\
\text { (wks) }\end{array}$ & $\begin{array}{l}\text { Birth weight } \\
\text { (g) }\end{array}$ & $\begin{array}{l}\text { APGAR } \\
\text { scores }^{*}\end{array}$ & CU findings & Neurodevelopment \\
\hline 101 & 41 & 3510 & $9 / 10 / 10$ & bilateral multiple cyst & speaking/language disorder \\
\hline 3672 & 39 & 3600 & $9 / 10 / 10$ & bilateral multiple cyst & delayed speaking development \\
\hline 2735 & 40 & 3680 & $9 / 9 / 9$ & ventricular enlargement & speaking/language disorder \\
\hline 1614 & 40 & 4270 & $9 / 10 / 10$ & ventricular asymmetry & speaking/language disorder \\
\hline 3048 & 41 & 3200 & $9 / 10 / 10$ & ventricular asymmetry & $A D(H) D$ \\
\hline 744 & 40 & 4230 & $8 / 10 / 10$ & $\begin{array}{l}\text { unilateral cyst in caudothalamic } \\
\text { groove }\end{array}$ & auditory perception disorder \\
\hline 4708 & 39 & 2890 & $8 / 9 / 9$ & ventricular enlargement & tic disorder \\
\hline 2144 & 41 & 3900 & $7 / 9 / 10$ & ventricular asymmetry & epilepsy \\
\hline 3845 & 40 & 4220 & $9 / 10 / 10$ & ventricular enlargement & Rolando epilepsy, AD(H)D \\
\hline 2546 & 40 & 3940 & $9 / 9 / 10$ & ventricular asymmetry & $A D(H) D$ \\
\hline 3466 & 40 & 3360 & $9 / 10 / 10$ & unilateral cyst in plexus & speaking disorder \\
\hline 3935 & 36 & 2960 & $9 / 10 / 10$ & unilateral cyst in plexus & delayed motor and speaking development \\
\hline 4899 & 39 & 4060 & $9 / 10 / 10$ & $\mathrm{IVH} \mathrm{I}^{\circ}$ & auditory perception disorder \\
\hline 5373 & 41 & 3810 & $8 / 9 / 10$ & unilateral cyst in septum pellucidum & $\begin{array}{l}\text { adaptive functioning emotional disorder/tic } \\
\text { disorder }\end{array}$ \\
\hline 3897 & 39 & 2825 & $8 / 9 / 10$ & unilateral cyst in plexus & delayed motor development \\
\hline
\end{tabular}

*APGAR scores were measured at $1 \mathrm{~min} / 5 \mathrm{~min} />5 \mathrm{~min}$ after birth; CU: cerebral ultrasound; AlUM: American Institute of Ultrasound in Medicine; NCU: neonatal care unit; APGAR score: assessment score for appearance, pulse, grimace, activity, and respiration of the neonate; $A D(H) D$ : attention deficit (and hyperactivity) disorder; IVH: intraventricular hemorrhage 
[8]. Multiple cysts, irrespective of location, can be associated with, for example, IVH and PVL. In addition, multiple cysts can lead to further malformations that have an impact on neurodevelopment [13]. In this study, among patients in group II with SCs that represented bilateral cysts, $11.8 \%(n=2 / 17)$ showed neurodevelopmental delay or speaking disorders; the cumulative incidence was $0.05 \%(n=2 / 3696)$ in all neonates without a CU indication or NCU admission. None of the named associated diseases or malformations were found.

PVL typically appears in very premature infants, but it is also detected on a $\mathrm{CU}$ or magnetic resonance imaging (MRI) at an unknown incidence in late preterm and term infants [13]. PVL grades 1 to 3 exhibit (1) an abnormally high signal intensity, (2) loss of periventricular white matter, or (3) a necrotic, diffuse component, which can lead to cysts, focal scars, and global delay in myelination. These features were correlated with cerebral palsy and cognitive or behavioral abnormalities [13, 14]. Among children with PVL grade 1, 56\% might also have minor motor problems. An association with West syndrome, which presents with relapsing seizures, increases the probability of disorders in brain development and psychomotor functioning [15]. In this study, PVL was not associated neurological impairment, and the cumulative incidence of PVL in group II was low (0.03\%).

In the general population, the incidence of partial or complete agenesis of the corpus callosum is 0.3 to $0.7 \%$, and it can be detected with CU [16]. The outcome of partial agenesis of the corpus callosum spans a large range, from mild behavioral problems to severe neurological disorders. Compared to complete agenesis, a partial agenesis is associated with a higher probability of combined genetic syndromes, aneuploidies, further cerebral malformations, inborn errors of metabolism, and extracerebral malformations [17-19]. .Compared to the intelligence of the general population, children with isolated corpus callosum agenesis have a higher probability of borderline intelligence and a lower probability of a Full Scale Intelligence Quotient $\geq 100$ [20]. In this study, we found a cumulative incidence with isolated corpus callosum agenesis of $0.03 \%$ in group II, similar to that reported by Wang et al. (0.08\%), but we found no correlation with neurological impairment [11].

$\mathrm{IVH} \geq \mathrm{II}^{\circ}$ can induce epileptic disorders, perceptual difficulties, cognitive deficiencies, and mental handicaps $[8$, 14, 21]. In particular, IVH can induce a worse outcome in full-term neonates than in preterm neonates [14]. Even a mild IVH alone can independently have an impact on neurological outcome, compared to no IVH [22]. .We found a $0.05 \%$ cumulative incidence of IVH in group II, which was lower than the incidences found in studies by Gover $(0.2 \%)$ and Wang et al. $(0.1 \%)$, and we found no association with neurological impairment [11, 12].
Our results suggested that it is not necessary to extend the recent $\mathrm{CU}$ guidelines for neonates without a $\mathrm{CU}$ indication. We found an existing, but low cumulative incidence of mild and significant anomalies; thus, we could not demonstrate a correlation between ultrasound findings and neurological follow-up. Neurological development disorders can be detected during the regular, continual pediatric check-ups in Germany. Nevertheless, neonatologists, follow-up-clinicians, parents, and teachers must be cautious in assessing infants with the named pathologies, because additional resources and sustained rehabilitation might be necessary to maximize the child's potential [20,22].

Our study findings emphasized the necessity of conducting CUs in cases specified in the recommended guidelines. Indeed, we found that all neonates $<30$ weeks GA had an indication for $\mathrm{CU}$; the cumulative incidence was $34.9 \%$ for a $\mathrm{CU}$ finding, and the probability was $20.9 \%$ for neurodevelopmental disorders. Among neonates with a NCU admission (7.7\%) the highest cumulative incidence for SCs was observed (2.8\% SCs), and of these, $16.4 \%$ displayed neurodevelopmental disorders. Almost every 5th neonate born at 30-34 weeks GA had a CU conspicuity. This finding was consistent with findings by Ballardini et al., who suggested that $\mathrm{CU}$ should be performed in all neonates born at $\leq 34$ weeks GA [9].

The major strength of this study was the large number of neonates $(n=5109)$ with data available from the population-based prospective cohort study, SNiP, including 3696 neonates without a CU indication The analysis included neonates at ages that ranged between 22 and 43 weeks GA, which exceeded the age ranges studied in previous investigations.

Although the SNiP database comprised 250 variables, data on follow-up were not included; consequently, those data were collected retrospectively. The major limitation of this study was the $25 \%$ loss to follow-up in the most interesting group of neonates, which had no indication for $\mathrm{CU}$. The nature of the retrospective study design for the follow up and reevaluation of sonographies impeded a detailed assessment of developmental disorders; therefore, we might have missed some neonates with conspicuities in the $\mathrm{CU}$ that later displayed developmental disorders, which is a limitation. The increased diagnostic accuracy of prenatal ultrasound in the last decade can also increase the amount of prenatal diagnosed abnormal cerebral findings in future. Furthermore, any unusual CU findings, including normal variations, were documented as conspicuities in the SNiP database. To eliminate normal variations and detect missed conspicuities, we performed a retrospective re-evaluation of the scanned $\mathrm{CU}$ images; this reevaluation might have resulted in some unrecognized pathologies. 


\section{Conclusion}

This study could not demonstrate either a probable association between ultrasound findings and neurological follow-ups or any advantage to performing an earlier diagnosis. Therefore, our data did not support an extension of current guidelines or the need for a general $\mathrm{CU}$ screening policy for all neonates. Nevertheless, the $\mathrm{CU}$ is helpful to detect brain abnormalities earlier. We recommend that $\mathrm{CU}$ should be performed in all neonates that conform to the recent recommendations of the AIUM, Leijser et al., Ment et al., and Ballardini et al. [3-5, 9] Further investigations of outcome are needed.

\section{Abbreviations \\ AIUM: American Institute of Ultrasound in Medicine; APGAR score: Assessment score for appearance, pulse, grimace, activity, and respiration of the neonate; CU: Cranial ultrasound; IVH: Intraventricular hemorrhage; MC: Mild conspicuity; NCU: Neonatal care unit; PVL: Periventricular leukomalacia; SC: Significant conspicuity; SNiP: Survey of Neonates in Pomerania; weeks GA: Weeks of gestational age}

\section{Acknowledgements}

This work is part of the Research Cooperation Community Medicine (RCC) of the University of Greifswald, Germany, which is funded by the Federal Ministry of Education and Research (grant no. ZZ 96030). The RCC encompasses several research projects that share data obtained through population-based studies (http://www.community-medicine.de). SNiP was supported by the German Federal Ministry for Education and Research (NBL3 program, grant 01 ZZ 0103), the Excellence Support Programme of the Federal State of Mecklenburg-Western-Pomerania (UG 07 034) "Genetics of frequent variants in INSIG2, their role in the pathogenesis of obesity and other disorders particularly in children", and the Epidemiological Study on Childhood Cancer and Malformations in the Vicinity of Nuclear Power Plants (Stsch 4493). The Medical Faculty of the Ernst Moritz Arndt University of Greifswald provided additional support in the form of a training grant to AL. We acknowledge support for the Article Processing Charge from the DFG (German Research Foundation, 393148499) and the Open Access Publication Fund of the University of Greifswald.

\section{Competeing interests}

The authors declare that they have no competing interests.

\section{Authors' contributions}

$J W$ and AEL were responsible for data collection and writing of the manuscript. $\mathrm{HB}, \mathrm{HA}$ and $\mathrm{MH}$ were responsible in data collection and quality assessment, GD and TI were responsible for the statically analysis, AEL originally conceived of the study and contributed to scientific interpretations and revise the manuscript. The authors declare that they have no competing interest. I confirm that all authors have read and approved submission of the manuscript and that material in the manuscript has not been published and is not being considered for publication elsewhere in whole or in part in any language or any form.

\section{Funding}

SNiP was supported by (a) the German Federal Ministry for Education and Research (NBL3 program, grant 01 ZZ 0103), (b) the Excellence Support Programme of the Federal State of Mecklenburg-Vorpommern (UG 07034 ) 'Genetics of frequent variants in INSIG2, their role in the pathogenesis of obesity and other disorders particularly in children' awarded to D. Rosskopf and J. P. Haas, (c) the Epidemiological Study on Childhood Cancer and Malformations in the Vicinity of Nuclear Power Plants (StSCH 4493). The funders had no role in study design, data collection and analysis, decision to publish, or preparation of the manuscript.

\section{Availability of data and materials}

The data of the SNIP-study is publicly available. This is a data repository where any researcher can register and find data dictionary as well as an online application tool for getting access to data. Upon an application by registered users, the Research Cooperation Community Medicine (RCC) of the
University of Greifswald, Germany, which is funded by the Federal Ministry of Education and Research (grant no. ZZ 96030) decides on granting access to the data based on scientific guidelines.

\section{Ethics approval and consent to participate}

Research based on patient-related data and human DNA is strictly regulated by German law. The collection of detailed personal data combined with sampling of biomaterials demands strict confidentiality. Our study complies with international guidelines of ethical research based on the Declaration of Helsinki. The study design was reviewed and approved by the Ethics Committee of the Board of Physicians Mecklenburg-Western Pomerania at the University of Greifswald. Eligible women were asked for written informed consent; in cases of legally minor mothers, i.e. aged $<18$ years, the additional signature of the newborn's legal caregiver was required. Written informed consent included data assessment in face-to-face interviews, selfadministered questionnaires and patient records. It also covered biosamples of blood. Furthermore, data storage of pseudonymized data was given informed consent to, as well as their analyses and publication.

\section{Consent for publication}

not applicable.

\section{Author details}

${ }^{1}$ Dept. of Neonatology \& Paediatric Intensive Care Medicine, University Greifswald, F.-Sauerbruchstr, 17475 Greifswald, Germany. ${ }^{2}$ Institute of Community Medicine, Div. of Health Care Epidemiology and Community Health, University Greifswald, Greifswald, Germany.

Received: 17 January 2019 Accepted: 20 November 2019

Published online: 05 December 2019

\section{References}

1. Fox TB. Sonography of the neonatal brain. Journal of Diagnostic Medical Sonography. 2009;25:331-48.

2. Fritz J, Polansky SM, O'Connor SC. Neonatal neurosonography. Semin Ultrasound CT MR. 2014:35:349-64.

3. Ment LR, Bada HS, Barnes P, et al. Practice parameter: neuroimaging of the neonate: report of the quality standards Subcommittee of the American Academy of neurology and the practice Committee of the Child Neurology Society. Neurology. 2002;58:1726-38.

4. Leijser LM. de Vries, Linda S, Cowan FM. Using cerebral ultrasound effectively in the newborn infant. Early hum. Dev. 2006;82:827-35.

5. American Institute of Ultrasound in Medicine (AIUM): American College of Radiology (ACR); Society of Radiologists in Ultrasound(SRU) Collaborators. AIUM practice guideline for the performance of neurosonography in neonates and infants. J Ultrasound Med. 2014;33(6):1103-10. https://doi.org/ 10.7863/ultra.33.6.1103

6. Ebner A, Thyrian JR, Lange A, et al. Survey of neonates in Pomerania (SNiP): a population-based birth study--objectives, design and population coverage. Paediatr Perinat Epidemiol. 2010;24:190-9.

7. Riccabona $M$, Schweintzger $G$, vonRohden $L$, et al. Documentation guideline: Documentation of Ultrasound of the Neurocranium (Standarddokumentation der Sonografie des kindlichen Neurokraniums), 2004

8. Fernandez Alvarez J. R, Amess PN. Gandhi RS et al Diagnostic value of subependymal pseudocysts and choroid plexus cysts on neonatal cerebral ultrasound: a meta-analysis Arch Dis Child Fetal Neonatal Ed. 2009;94:F4436.

9. Ballardini E, Tarocco A, Baldan A, et al. Universal cranial ultrasound screening in preterm infants with gestational age 33-36 weeks. A retrospective analysis of 724 newborns. Pediatr. Neurol. 2014;51:790-4.

10. Ballardini E, Tarocco A, Rosignoli C, et al. Universal head ultrasound screening in full-term neonates: a retrospective analysis of 6771 infants. Pediatr Neurol. 2017;71:14-7.

11. Wang LW, Huang CC, Yeh TF. Major brain lesions detected on sonographic screening of apparently normal term neonates. Neuroradiology. 2004;46: 368-73.

12. Gover A, Bader D, Weinger-Abend M, et al. Head ultrasonograhy as a screening tool in apparently healthy asymptomatic term neonates. Isr Med Assoc J. 2011 Jan;13(1):9-13. 
13. Kinney HC. The near-term (late preterm) human brain and risk for periventricular leukomalacia: a review. Semin Perinatol. 2006;30:81-8

14. Volpe JJ, editor. Neurology of the newborn. 5th ed. Philadelphia: Saunders/ Elsevier; 2008.

15. Imamura $\mathrm{T}$, Ariga $\mathrm{H}$, Kaneko $\mathrm{M}$, et al. Neurodevelopmental outcomes of children with periventricular leukomalacia. Pediatrics and neonatology. 2013;54:367-72

16. Volpe P, Paladini D, Resta M, et al. Characteristics, associations and outcome of partial agenesis of the corpus callosum in the fetus. Ultrasound Obstet Gynecol. 2006;27:509-16.

17. Kidron D, Shapira D, Ben Sira L, et al. Agenesis of the corpus callosum. An autopsy study in fetuses. Virchows Arch. 2016;468(2):219-30. https://doi.org/ 10.1007/s00428-015-1872-y

18. Ozyüncü O, Yazıcıoğlu A, Turğal M. Antenatal diagnosis and outcome of agenesis of corpus callosum: a retrospective review of 33 cases. J Turk Ger Gynecol Assoc. 2014;15:18-21.

19. Moutard M-L, Kieffer V, Feingold J, et al. Agenesis of corpus callosum: prenatal diagnosis and prognosis. Childs Nerv Syst. 2003;19:471-6.

20. Moutard M-L, Kieffer V, Feingold J, et al. Isolated corpus callosum agenesis: a ten-year follow-up after prenatal diagnosis (how are the children without corpus callosum at 10 years of age?). Prenat Diagn. 2012;32:277-83.

21. Brouwer AJ, Groenendaal F, Benders MJNL, et al. Early and late complications of germinal matrix-intraventricular haemorrhage in the preterm infant: what is new? Neonatology. 2014;106:296-303.

22. Mukerji A, Shah V, Shah PS. Periventricular/Intraventricular hemorrhage and neurodevelopmental outcomes: a meta-analysis. Pediatrics. 2015;136:1132-43.

\section{Publisher's Note}

Springer Nature remains neutral with regard to jurisdictional claims in published maps and institutional affiliations.

Ready to submit your research? Choose BMC and benefit from:

- fast, convenient online submission

- thorough peer review by experienced researchers in your field

- rapid publication on acceptance

- support for research data, including large and complex data types

- gold Open Access which fosters wider collaboration and increased citations

- maximum visibility for your research: over $100 \mathrm{M}$ website views per year

At $\mathrm{BMC}$, research is always in progress.

Learn more biomedcentral.com/submissions 